\title{
La educación en clave audiovisual y multipantalla
}

\section{(Audiovisual and Multiscreen Education)}

\author{
Ignacio Aguaded \\ Universidad de Huelva \\ Miguel-Angel Ortiz-Sobrino \\ Universidad Complutense de Madrid \\ Coordinadores del Monográfico
}

DOI: $\underline{\text { https://doi.org/10.5944/ried.25.1.31454 }}$

\section{Cómo referenciar este artículo:}

Aguaded, I., y Ortiz-Sobrino, M. A. (2022). La educación en clave audiovisual y multipantalla. RIED. Revista Iberoamericana de Educación a Distancia, 25(1), pp. 31-39. https://doi.org/10.5944/ried.25.1.31454

\section{Resumen}

El potencial educativo de los medios audiovisuales ha sido objeto de estudio, sobre todo, a partir del nacimiento de la televisión a mediados del siglo pasado. Diferentes investigadores han reparado en la conveniencia de su uso como herramienta educativa, dada su capacidad para facilitar a los alumnos la comprensión de los contenidos educativos a través de las imágenes, los sonidos y los gráficos que incorporan como lenguaje. Durante muchos años, el foco de las investigaciones sobre este tema se centró en los medios de comunicación social convencionales: el cine, la radio y, especialmente, la televisión. Sin embargo, la definitiva implementación de las TIC en los procesos educativos, la aparición de la denominada sociedad multipantalla y las nuevas plataformas de distribución de contenidos, han originado un nuevo entramado mediático en el que los media tradicionales van perdiendo protagonismo en favor de los nuevos dispositivos móviles, de la web social y de los denominados social media. Este nuevo escenario ha derivado en un cambio sustancial en las metodologías de enseñanza y una profunda transformación del rol de profesores y alumnos. El protagonismo de los contenidos audiovisuales educativos, sus formatos adaptados para dispositivos móviles y la consolidación de la web como sistema de aprendizaje reglado o no formal son realidades que, aunque ya estaban consolidadas, la situación de pandemia por COVID-19 las ha reforzado. Este monográfico incorpora el análisis de varios investigadores sobre las posibilidades educativas del sonido, el vídeo y la imagen en el actual entramado digital.

Palabras clave: medios audiovisuales; sociedad multipantalla; educación; recursos educativos audiovisuales; plataformas digitales educativas. 


\begin{abstract}
The educational potential of audiovisual media has been the object of study, especially since the birth of television in the middle of the last century. Researchers have noticed the convenience of its use as an educational tool, given its ability to facilitate students' understanding of educational content through the images, sounds and graphics that they incorporate as language. For many years the focus of research on this topic was on conventional social media: cinema, radio and, above all, television. However, the definitive implementation of ICTs in educational processes, the emergence of the so-called multiscreen society and the new content distribution platforms have created a new media framework where traditional media are losing prominence in favor of new devices. mobile phones, the social web and social media. This new scenario has led to a substantial change in teaching methodologies and a profound transformation of the role of teachers and students. The prominence of educational audiovisual content, its formats adapted for mobile devices and the consolidation of the web as a formal or non-formal learning system are realities that have been reinforced by the COVID-19 pandemic. This monograph incorporates the perspectives of various researchers on the educational possibilities of sound, video and image in the current digital framework.
\end{abstract}

Keywords: audiovisual media; multiscreen society; education; audiovisual educational resources; educational digital platforms.

La radio, el cine y, especialmente la televisión, fueron los ejes centrales del discurso sobre el potencial educativo de los medios audiovisuales hasta la primera década de este siglo (Cabero, 2007; Peralta Ferreyra, 2005). No es casual el interés de los investigadores por su papel en el contexto educativo a lo largo de la centuria. Algunos investigadores, como Vital-Rumebe et al. (2021), defienden el uso de los medios audiovisuales como herramienta educativa basándose en su capacidad para facilitar a los alumnos la comprensión a través de las imágenes, sonidos y gráficos que incorporan como lenguaje. El empleo de películas y vídeos como material de apoyo educativo lograron captar la atención del estudiante, produciendo en ellos un sentimiento de empatía. Esto, combinado con la asesoría de los profesores en el aula, hizo que el proceso de aprendizaje fuera más eficiente. Hasta tal punto, que desde la literatura científica se ha puesto de manifiesto en varias ocasiones el esfuerzo de las Administraciones para incorporar de forma generalizada los recursos audiovisuales como herramientas de apoyo para el aprendizaje (Aguaded y Tirado, 2010; HidalgoNavarrete y Aliaga-Zegarra, 2020).

En España, ha transcurrido casi medio siglo desde el comienzo de las primeras emisiones educativas de la Universidad Nacional de Educación a Distancia (UNED), a través del entonces denominado "Tercer Programa" de Radio Nacional de España. Corría entonces el año 1973. Unos años antes, en 1967, la emisora Radio ECCA comenzó a emitir contenidos radiofónicos como apoyo educativo. Después llegaría, en 2011, la consolidación de las radios universitarias españolas (Ortiz-Sobrino et al., 
2021). En el camino quedaron experiencias de programas radiofónicos dedicados a la universidad, como el espacio "Tiempo de Universidad "de Radio 3, en la década de los 80, o "Mundo Universitario" de Radio 5, en los años 90. En otros países europeos, como Francia o Reino Unido, las experiencias se remontan a la década de los 6o. Por su parte, en América se constatan experiencias radiofónicas centenarias de radio educativa, como las emisiones de la Radio Universitaria de la Universidad de la Plata, en Argentina.

Algo parecido ha sucedido con la televisión, y en menor medida con el cine. En España, la televisión pública, por ejemplo, viene emitiendo los programas televisivos de la Universidad Nacional de Educación a Distancia (UNED) y, en paralelo, su propio espacio de contenidos educativos y de divulgación, como el programa "La aventura del saber". El recorrido seguido en otros países ha sido muy parecido, tanto en Europa como en América. Así, en Italia se iniciaron las clases por televisión en los años 60, mientras que en México también se implantó en esa década el sistema de "Telesecundarias", como una política educativa de Estado. Más tarde, a finales de los años 80, también aparecieron los primeros canales de pago con fines educativos, como Discovery Channel en 1985 (Vital-Rumebe et al., 2021).

Sin embargo, el nuevo escenario digital ha transformado el rol de los media tradicionales. Desde la llegada de Internet a España en los años 9o, las tecnologías digitales han demostrado la diversidad de aplicaciones en múltiples campos, entre ellos en la educación formal y en el ámbito familiar (Aguaded y Contin, 2002; Jiménez-Morales et al., 2020). La irrupción de lo que ha venido a denominarse la sociedad multipantalla, como consecuencia de la generalización del uso de los smartphones, los sucesivos desarrollos de la web 2.o y la adopción por parte de la comunidad educativa de las nuevas plataformas digitales asociadas a las TIC, ha transformado ese discurso. Hasta tal punto, que aquellos mass media tradicionales que reclamaban su protagonismo en el sistema educativo durante la segunda mitad del siglo XX, han ido compartiendo su protagonismo con las nuevas pantallas, sobre todo a partir del desarrollo de los social media en la segunda década del actual milenio (Ortega-Mohedano y Pinto-Hernández, 2021). La cohabitación de este nuevo escenario mediático con los tradicionales medios de comunicación que desde el pasado siglo habían sido referencia como soportes fundamentales para la educación formal y no formal, ha dado paso a una nueva praxis en la utilización de los recursos audiovisuales como apoyo a la educación.

En este sentido, Hidalgo-Navarrete y Aliaga-Zegarra (2020) han puesto de manifiesto cómo, en ese escenario multipantalla, el acceso a recursos audiovisuales se ha hecho ubicuo a través de los dispositivos móviles que los alumnos poseen. Las tabletas y los smartphones inteligentes se convierten a diario en pequeñas salas de cine o televisiones portátiles en las manos del alumnado. No solo posibilitan su acceso a los contenidos didácticos audiovisuales, sino también su participación de forma colaborativa en la producción. 
La importancia de este nuevo entorno tecnológico está transformando el aula y las metodologías educativas tradicionales. Tanto, que se ha convertido en un fenómeno de enorme interés para la comunidad científica. Prendes-Espinosa y Cerdán-Cartagena (2021), entre otros, han abordado la revisión analítica de algunas experiencias didácticas relacionadas con la aplicación de las TIC en elámbito educativo -realidad aumentada, videojuegos y apps educativas, entre otras- que ofrecen un gran abanico de posibilidades de aplicación, tanto en entornos educativos formales como no formales. Su principal conclusión fue la gran cantidad de experiencias que recoge la literatura científica sobre este tema y el interés creciente que las TIC y los medios audiovisuales siguen despertando entre la comunidad educativa: fundamentalmente, a raíz del consumo multipantalla entre los estudiantes. En esa misma línea, García-Martín y García-Martín (2021) han analizado el uso de quince herramientas digitales para la docencia entre las que se contemplan diferentes plataformas educativas, los blogs, las wikis, las herramientas de gamificación, las herramientas para crear contenido colaborativo e interactivo, así como para editar vídeos y para programar contenidos.

Este nuevo panorama ha afectado especialmente al papel de uno de los medios convencionales de referencia para la educación, la televisión, en la medida en la que el nuevo panorama tecnológico le ha obligado a redefinirse. En este sentido, Marta-Lazo et al. (2020) recogen la posición de Aguaded sobre el nuevo papel de la televisión en esta sociedad multipantalla. Sostiene este investigador que el papel educativo de la televisión debe centrarse ahora en superar la vertiente escolar y atender la necesaria transversalidad de contenidos e incidir en los ejes transversales de un currículum de formación ciudadana. La educación no es solo un contenedor de conceptos, sino un aprendizaje para la vida en sociedad en el que la televisión tiene mucho que aportar desde un punto de vista educativo-cultural. La televisión global (online, multimedia, multicanales, multi-dispositivos, a la carta...) no tiene marcha atrás, y solo en la medida que la televisión tradicional se sume a esta revolución tecnológica tendrá garantizado su espacio en el currículum educativo (Aguaded et al., 2015; Marta-Lazo et al., 2020).

El modelo social basado en la Web social y la universalización de las TIC que permiten compartir, crear y publicar contenidos es algo ya contrastado. Hoy las diferentes plataformas tecnológicas y los social media son espacios que se entrecruzan configurando aulas multipantalla interconectadas. El fenómeno podcast, la webcaster, los recursos audiovisuales educativos virtuales o la producción de contenidos audiovisuales colaborativos son ya realidades que marcan la tendencia en los binomios Educación-TIC y Educación-Contenidos digitales.

Sin embargo, el punto de inflexión en el papel de los contenidos educativos audiovisuales se ha producido en el último año. La experiencia del confinamiento por la COVID-19 ha marcado un punto de inflexión para la educación. No solo en la denominada formación reglada, sino también en lo que se ha venido a denominar lifelong learning (aprendizaje a lo largo de la vida o formación continua). 
Como consecuencia de la pandemia mundial en marzo de 2020, las instituciones de enseñanza españolas se vieron obligadas a la suspensión de las actividades docentes presenciales y adaptarlas a un formato virtual que ha supuesto un rediseño del sistema de aprendizaje habitual y de los sistemas de evaluación en todas las etapas educativas (García-Martín y García-Martín, 2021). El cierre de los centros educativos provocó la sustitución de la enseñanza presencial por otros contenidos adaptados a formatos online y en lenguaje audiovisual. Las nuevas metodologías de enseñanza como el e-learning, el blended-learning, flipped classroom o el x-learning- utilizan ahora el podcast y el vídeo en sus diferentes formatos y tratamientos como elementos estructurales para su implementación (García-Marín y Aparici, 2020). Se trata de modelos educativos que la pandemia de COVID-19 está acelerando y consolidando, sin lugar a duda.

En los capítulos que integran este monográfico se incorpora el análisis de varios investigadores sobre las posibilidades educativas del sonido, el vídeo y la imagen en el actual entramado digital. En primer lugar, presentamos un artículo que aborda la producción de contenidos audiovisuales en el ámbito educativo, analizando las propuestas desarrolladas en diversos contextos de Iberoamérica. En el trabajo de Toscano, Aguaded, Manotas y Farias, titulado "Producción audiovisual universitaria: espacios de innovación docente en Iberoamérica", reflexionan sobre la necesidad de promover el acompañamiento pedagógico para lograr un mayor aprovechamiento de los recursos y los espacios especializados de producción, así como para alcanzar el empoderamiento de los agentes educativos implicados en los procesos de enseñanzaaprendizaje.

Como vinculación a este trabajo, se presenta un segundo artículo en el que se evalúan precisamente las perspectivas docentes para la producción de contenido audiovisual a través de los MOOC (Massive Open Online Courses). De este modo, Freitas y Paredes en "Desafíos de la producción multimedia en los MOOC. Estudio de caso interpretativo sobre las perspectivas docentes" analizan las experiencias innovadoras desarrolladas en estos entornos virtuales en la Universidad Autónoma de Madrid (UAM), así como la implicación del profesorado y las principales oportunidades y dificultades presentes en el desarrollo de este tipo de proyectos.

$\mathrm{Al}$ igual que en el caso anterior, el tercer artículo de Caerols, Sindorenjo y Osuna "Los MOOC en la formación continua y especializada: ¿nuevas narrativas audiovisuales? Mitos y retos" aborda la temática de los MOOC mediante la realización de una revisión de literatura acerca del surgimiento de estos cursos y el análisis de sus características metodológicas y narrativas. Desde el enfoque teórico de la Educomunicación y el fenómeno transliteracy, se plantea la necesidad de corroborar si los MOOC realmente se desarrollan con planteamientos metodológicos innovadores, o si continúan promoviendo procesos de enseñanza-aprendizaje basados en modelos educativos tradicionales.

El cuarto artículo, "Aprendizaje multimedia y transferencia de conocimiento en una plataforma digital. Estudio de caso de Entremedios", de Marta, Gabelas, Nogales 
y Badillo, presenta esta plataforma, que favorece también el aprendizaje multimedia y la transferencia de conocimiento a través de un entorno virtual, destacando sus posibilidades mediante las TRIC (Tecnologías de la Relación, Información y Comunicación) en los procesos educomunicativos para el desarrollo del pensamiento crítico y reflexivo, destacando aquellos aspectos mejorables que podrían optimizar la usabilidad e interacción del alumnado.

Asimismo, cabe destacar que las estrategias desarrolladas por las instituciones educativas para la formación docente se han visto incrementadas durante la COVID-19. Concretamente, en el quinto manuscrito, Sánchez González, Miró, Ruiz y Cebrián de la Serna analizan la iniciativa de una universidad para la capacitación del profesorado y el desarrollo de su competencia digital a través de seminarios online. En el trabajo "Evaluación de programas online de capacitación docente sobre innovación y competencias digitales durante la Covid-19: \#webinaranonimizado" se centran en apoyar y dar respuesta a las necesidades formativas de los docentes a raíz de las medidas restrictivas derivadas de la situación de emergencia provocada por la pandemia y la consecuente e inesperada virtualización de las clases.

Estas consecuencias e imprevistas necesidades educativas han afectado a diversos colectivos, como es el caso de las personas con discapacidad, tal y como se pone de manifiesto en la sexta investigación, que aborda los retos que los diferentes agentes educativos de un centro ocupacional tuvieron que afrontar ante esta nueva realidad. En este sentido, Bonilla y Sánchez-Calero en "Inclusión educativa en tiempos de COVID-19: Uso de redes sociales en personas con discapacidad intelectual" analizan los usos, beneficios y dificultades que encontraron ante el uso de las redes sociales, así como los cambios que el confinamiento supuso en sus experiencias educativas, sociales y comunicativas.

En el siguiente trabajo, "Educomunicación, webradio y educación no formal en un contexto Erasmus+: La experiencia de Europa on air", González-Pérez, OrtizSobrino y López-Vidales exponen la importancia y las posibilidades que ofrece el uso de redes sociales y la webradio, en este caso en prácticas de educación no formal. Este séptimo artículo presenta la experiencia de un proyecto Erasmus+ que tiene como finalidad la mejora de las competencias comunicativas, lingüísticas y las relacionadas con la interculturalidad, la ciudadanía y la diversidad.

Del mismo modo, la octava aportación de González-Conde, titulada "Didáctica del podcast en el programa PMAR. Una experiencia de aula en la Comunidad de Madrid", aborda una experiencia de aula que también tiene como objetivo desarrollar las competencias sociolingüísticas, emocionales y cognitivas del alumnado de educación secundaria a través del uso didáctico del podcast mediante un enfoque de carácter lúdico e innovador del proceso de enseñanza-aprendizaje. Esta investigación de acción participativa y dialógica profundiza en los resultados obtenidos a partir del proceso práctico de este proyecto educativo.

Además de la radio y los podcasts, otro de los medios por excelencia que puede emplearse con fines educativos es la televisión. En este noveno artículo "La televisión 
educativa en España: la oferta de Atresmedia, Mediaset y RTVE”, de RodríguezCastro, López-Cepeda y Soengas, se realiza un recorrido histórico de la evolución de la oferta educativa de la televisión en España, analizando la programación de tres grandes grupos mediáticos: Atresmedia, Mediaset y RTVE, con el fin de identificar las principales características de los programas educativos emitidos a nivel nacional y el espacio dedicado a la emisión de contenidos educativos.

\section{REFERENCIAS}

Aguaded, I., y Contin, S. (2002). Jóvenes, aulas y medios de comunicación. La Crujía.

Aguaded, I., y Tirado, R. (2010). Ordenadores en los pupitres: Informática y telemática en el proceso de enseñanza-aprendizaje en los centros TIC de Andalucía. Pixel-Bit, 36, 5-28.

Aguaded, I., Marín-Gutiérrez, I., y DíazPareja, E. (2015). La alfabetización mediática entre estudiantes de primaria y secundaria en Andalucía (España). RIED. Revista Iberoamericana de Educación a Distancia, 18(2), 275-298. https://doi. org/10.5944/ried.18.2.13407

Cabero, J. (2007). La televisión educativa: aspectos a contemplar para su integración curricular. Anales de la Universidad Metropolitana, 7(1).

García-Marín, D., y Aparici, R. (2020). Voces domesticadas y falsa participación: Anatomía de la interacción en el podcasting transmedia. Comunicar, 63, 97-107. https://doi.org/10.3916/C632020-09

García-Martín, J., y García-Martín, S. (2021). Uso de herramientas digitales para la docencia en España durante la pandemia por COVID-19. Revista Española de Educación Comparada, 38 (extra 2021), 151-173.

Hidalgo Navarrete, J. y Aliaga Zegarra, S. E. (2020). Análisis de las estrategias didácticas para el diseño, selección, producción, utilización y validación de recursos educativos audiovisuales interactivos en una institución educativa. estudio inicial. Revista Electrónica de Investigación y Docencia (REID). 23 (feb. 2020), 79-98. https://doi. org/10.17561/10.17561/reid.n23.5.

Jiménez-Morales, M., Montaña, M., y Medina -Bravo. P. (2020). Uso infantil de dispositivos móviles: Influencia del nivel socioeducativo materno. Comunicar, 64, https://doi.org/10.5944/ reec.38.2021.27816

Marta-Lazo, C., Ortiz-Sobrino, M. A., GómezPatiño, M., y González, J.C. (2020). COVID-19 y medios de comunicación: Voces para un análisis crítico. Sindéresis. Ortega-Mohedano, F., y Pinto-Hernández, F. (2021). Predicción del bienestar sobre el uso de pantallas inteligentes de los niños. Comunicar, 66, 119-128. https://doi. org/10.3916/C66-2021-10

Ortiz-Sobrino, M. Ángel, Marta-Lazo, C, y Contreras-Pulido, P. (2021). Competencias profesionales para la gestión de emisoras universitarias en España. Cuadernos.Info, 48, 189-214. https://doi.org/10.7764/cdi.48.27667

Peralta-Ferreyra, I. (2005). El televisor: la pantalla estrella. Comunicar, 25, 1, 193195. https://doi.org/10.3916/C25-2005$\underline{026}$

Prendes-Espinosa, M. P., y CerdánCartagena, F. (2021). Tecnologías avanzadas para afrontar el reto de la innovación educativa. RIED. Revista Iberoamericana de Educación a Distancia, 24(1), 35-53. https://doi. org/10.5944/ried.24.1.28415 
Vital-Rumebe, G., Ontiveros-Moreno; Guerra-Rojas, C., y Gutiérrez-Rocha, A. (2021). Videolearning: aprendizaje y educación a través de medios audiovisuales, desde una perspectiva histórica y contemporánea. Revista Panamericana de Pedagogía, 32, 216227.

\section{PERFIL ACADÉMICO Y PROFESIONAL DE LOS AUTORES}

Ignacio Aguaded. Catedrático de Universidad del Departamento de Educación de la Universidad de Huelva (España). Presidente del Grupo Comunicar, colectivo veterano en España en Educomunicación, y Editor Jefe de la reconocida revista científica 'Comunicar' (indexada en JCR-Q1, Scopus-Q1, top 1\% mundial...). Es además Investigador Principal (IP) del Grupo de Investigación ‘Ágora', con múltiples investigaciones nacionales e internacionales. Ha dirigido múltiples trabajos de investigación y más de 40 tesis doctorales. Es Director del Máster Internacional Interuniversitario de Comunicación y Educación Audiovisual (UNIA/UHU) y Director del Programa de Doctorado Interuniversitario en Comunicación (US, UMA, UCA y UHU). Actualmente es Presidente de la Red Internacional Euroamericana de Investigadores "Alfamed" compuesta por 18 países. Premio de Mejor Investigador de la Universidad de Huelva 2015 en Ciencias Sociales. https://orcid.org/0000-00020229-1118

E-mail: aguaded@uhu.es

\section{DIRECCIÓN DEL AUTOR \\ Universidad de Huelva \\ Facultad de Ciencias de la Educación. \\ 21071 Huelva (España).}

Miguel-Angel Ortiz-Sobrino. Profesor Titular en el Departamento de Periodismo y Nuevos Medios de la Universidad Complutense de Madrid. Doctor en Ciencias de la Información. Licenciado en Periodismo, Licenciado en Ciencias de la Imagen visual y auditiva y Diplomado Investigación de Medios por AIMC. Es Director del Master en Realización y Diseño de Programas y Formatos de Televisión y Director del Master en Comunicación Institucional Marketing y Eventos, ambos de la Universidad Complutense de Madrid. Es profesor permanente en el Programa de Periodismo y Comunicación Audiovisual de la UCM. Vinculado a RTVE desde 1977 hasta 2009, donde ha desempeñado, entre otros, los cargos de Jefe de Programas de Radio 5, Subdirector de Emisoras Territoriales de RNE y Subdirector de Planificación de Programas de RNE. Entre 2000 y 2009 dirigió el Instituto Oficial de Radio y Televisión. También ha sido Director Adjunto de Radio Televisión Madrid y Jefe de Programas de Onda Madrid. https://orcid.org/0000-0003-0103-9524 E-mail: maortiz@ucm.es 
DIRECCIÓN DEL AUTOR

Universidad Complutense de Madrid

Facultad de Ciencias de Ciencias de la Información.

28040 Madrid (España).

Fecha de recepción del artículo: 06/09/2021

Fecha de aceptación del artículo: 09/09/2021

Fecha de aprobación para maquetación: 20/09/2021 